\title{
A systematic literature review of health state utility values in head and neck cancer
}

Michela Meregaglia ${ }^{1,2^{*}}$ (i) and John Cairns $s^{1,3}$

\begin{abstract}
Background: Health state utility values (HSUVs) are essential parameters in model-based economic evaluations. This study systematically identifies HSUVs in head and neck cancer and provides guidance for selecting them from a growing body of health-related quality of life studies.

Methods: We systematically reviewed the published literature by searching PubMed, EMBASE and The Cochrane Library using a pre-defined combination of keywords. The Tufts Cost-Effectiveness Analysis Registry and the School of Health and Related Research Health Utilities Database (ScHARRHUD) specifically containing health utilities were also queried, in addition to the Health Economics Research Centre database of mapping studies. Studies were considered for inclusion if reporting original HSUVs assessed using established techniques. The characteristics of each study including country, design, sample size, cancer subsite addressed and demographics of responders were summarized narratively using a data extraction form. Quality scoring and critical appraisal of the included studies were performed based on published recommendations.
\end{abstract}

Results: Of a total 1048 records identified by the search, 28 studies qualified for data extraction and 346 unique HSUVs were retrieved from them. HSUVs were estimated using direct methods (e.g. standard gamble; $n=10$ studies), multi-attribute utility instruments (MAUIs; $n=13$ ) and mapping techniques $(n=3)$; two studies adopted both direct and indirect approaches. Within the MAUIs, the EuroQol 5-dimension questionnaire (EQ-5D) was the most frequently used $(n=11)$, followed by the Health Utility Index Mark $3(H U I 3 ; n=2)$, the 15D $(n=2)$ and the Short Form-Six Dimension (SF-6D; $n=1$ ). Different methods and types of responders (i.e. patients, healthy subjects, clinical experts) influenced the magnitude of HSUVs for comparable health states. Only one mapping study developed an original algorithm using head and neck cancer data. The identified studies were considered of intermediate quality.

Discussion: This review provides a dataset of HSUVs systematically retrieved from published studies in head and neck cancer. There is currently a lack of research for some disease phases including recurrent and metastatic cancer, and treatment-related complications. In selecting HSUVs for cost-effectiveness modeling purposes, preference should be given to EQ-5D utility values; however, mapping to EQ-5D is a potentially valuable technique that should be further developed in this cancer population.

Keywords: Health state utility values (HSUVs), Health-related quality of life (HRQOL), Head and neck cancer (HNC), Multi-attribute utility instruments (MAUIs), Standard gamble (SG), Time trade-off (TTO), Systematic literature review

\footnotetext{
* Correspondence: michela.meregaglia@unibocconi.it

'Department of Health Services Research and Policy, Faculty of Public Health

and Policy, London School of Hygiene and Tropical Medicine, 15-17

Tavistock Place, London WC1H 9SH, UK

${ }^{2}$ CeRGAS (Research Centre on Health and Social Care Management), Bocconi

University, Via Roentgen 1, 20136 Milan, Italy

Full list of author information is available at the end of the article
}

(c) The Author(s). 2017 Open Access This article is distributed under the terms of the Creative Commons Attribution 4.0 International License (http://creativecommons.org/licenses/by/4.0/), which permits unrestricted use, distribution, and reproduction in any medium, provided you give appropriate credit to the original author(s) and the source, provide a link to the Creative Commons license, and indicate if changes were made. The Creative Commons Public Domain Dedication waiver (http://creativecommons.org/publicdomain/zero/1.0/) applies to the data made available in this article, unless otherwise stated. 


\section{Background}

Cost-utility models are increasingly used to establish whether the cost of a new treatment is justified in terms of health gains. This approach usually adopts the quality-adjusted life year (QALY) as a measure of health effectiveness. According to Neumann et al., the QALY corresponds to the time spent in a series of qualityweighted health states, where the weights represent the desirability of living in that state [1]. The basic idea is that individuals move through health states over time and that each health state has a preference weight attached to it [2], also known as a health state utility value (HSUV). Thus, the HSUV can be interpreted as the strength of preference for a given health state on a cardinal scale anchored at 0 ('death') and 1 ('full health'), with some instruments also allowing for negative values representing states worse than death [3]. Therefore, QALYs are obtained by summing-up the products of the time spent in each health state and its corresponding preference-based value [4].

HSUVs can be estimated in a variety of ways including direct methods, multi-attribute utility instruments (MAUIs), mapping functions and expert opinion. The most common ways of eliciting HSUVs directly are gambling with respect to a hypothetical treatment that may result in perfect health or death (standard gamble, SG) or trading-off part of future life for a shorter time in perfect health (time trade-off, TTO) [5]. A further, simpler option is to use a visual analog scale (VAS), also known as rating scale, which provides an immediate valuation of the current (or a hypothetical) health state on a graduated scale, usually ranging between 0 and 100. This technique is generally considered to be methodologically inferior to choice tasks such as SG and TTO, which incorporate some extra information about the individual risk attitude [4]; VAS scores, indeed, are elicited in a choice-less context, and thus do not required respondents to make trade-offs within their utility function [6]. Moreover, the rating scales are well-known to present measurement biases such as context bias, spacing-out bias, and end-aversion bias [4,7]. Additionally, there is now consensus that health-related quality of life (HRQoL) is a multi-dimensional concept, which includes domains related to physical, mental, emotional, and social functioning that are difficult to measure on a single scale [8].

Direct measurement of health utility through SG or TTO can be complicated and time-consuming and lead to incomparable results across the studies due to arbitrary health state descriptions (also called 'vignettes') $[9,10]$. Consequently, in recent years, HSUVs have been increasingly estimated indirectly using multi-attribute utility instruments (MAUIs). These tools are formed of a generic HRQoL questionnaire and an accompanying formula or set of weights (or "tariffs") elicited from a sample of the general population for converting responses into HSUVs; thus, the utility measure can be considered as a preference-based evaluation of a given health state described by the dimensions of the tool $[11,12]$. The National Institute for Health and Care Excellence (NICE) and the European Network for Health Technology Assessment (EUnetHTA) recommend the EuroQol 5-dimension questionnaire (EQ-5D) (https://euroqol.org) [13, 14]. Accordingly, the TTO with a 10-year time horizon is the most frequently used approach among the direct techniques, because of greater comparability with the method used to develop the EQ-5D scoring algorithm [15]. The other generic MAUIs mostly adopted in the literature [11] are the Health Utility Index (HUI mark 2, HUI2 or mark 3, HUI3) [16], the Short Form-6-dimension (SF-6D) questionnaire derived from the 36-item Short Form Survey (SF-36) (https://www.sheffield.ac.uk/scharr/sections/ heds/mvh/sf-6d), the 15D (www.15d-instrument.net), the Quality of Wellbeing (QWB) index [17] and the Assessment of Quality of Life (AQoL) instruments [18].

In many situations, clinical studies neither administer preference-based MAUIs nor elicit HSUVs directly, but collect instead disease-specific HRQoL data or other clinical measures that are not associated with a preference-based scoring system; thus, QALY calculation from these studies is not possible. As a second-best solution, "mapping" or "cross-walking" has been developed to predict HSUVs from non-preference-based scores, provided that a statistical relationship can be established between the two instruments and, sometimes, allowing for the mediating effect of demographic and clinical characteristics [19].

This study focuses on HSUVs in head and neck cancer (HNC). Patients with HNC often undergo several rounds of treatment during which they experience acute toxicity and other side effects, such as loss of verbal abilities, difficulties in swallowing, and considerable pain [20]. This HRQoL impairment may continue long after treatment through persistent functional deficits, physical disfigurement, psychological distress, and recurrent disease. There is an extensive HRQoL literature in $\mathrm{HNC}$, although mainly comprised of disease-specific, nonpreference based data unsuitable for cost-utility comparisons. Due to the paucity of HSUVs for some health states in HNC, some previous cost-effectiveness analyses $[21,22]$ relied on values calculated for other cancers (such as breast or lung) to populate their models with utility parameters. A systematic review published in 2006 [23] identified eight studies providing utility values in HNC elicited through VAS, TTO or SG. Our study extends the collection of utility values related to this medical condition by systematically reviewing the studies 
published to date. This paper considers for inclusion studies of any design in which utility values in HNC were:

- directly elicited using standard techniques such as TTO or SG either in patient-based studies or in the general population;

- calculated indirectly from patient's responses to generic MAUIs (e.g. EQ-5D) through a set of tooland country-specific preference weights;

- predicted from non-preference based HRQoL instruments using mapping algorithms.

The Preferred Reporting Items for Systematic Reviews and Meta-Analyses (PRISMA) statement [24] is not entirely applicable to systematic reviews of HSUVs [25], since the standard Population, Intervention, Comparator, and Outcome (PICO) elements do not provide a useful framework for identifying utility values for health states that are not necessarily attached to a given intervention [26]. Thus, in this study we follow the recommendations provided by Papaioannou et al. [26]. The ultimate objective is to generate a database of HSUVs that might be useful to populate future cost-utility studies of interventions in HNC. In addition, we critically appraise the included studies by highlighting a few elements that should be considered when selecting utility parameters for modeling.

\section{Methods}

A systematic literature search was carried out of the PubMed, EMBASE and Cochrane Library databases for studies published from 2000 until the end of 2016 using a range of free-text terms in title/abstract (Fig. 1). Since Medical Subject Headings (MeSH) terms provide little

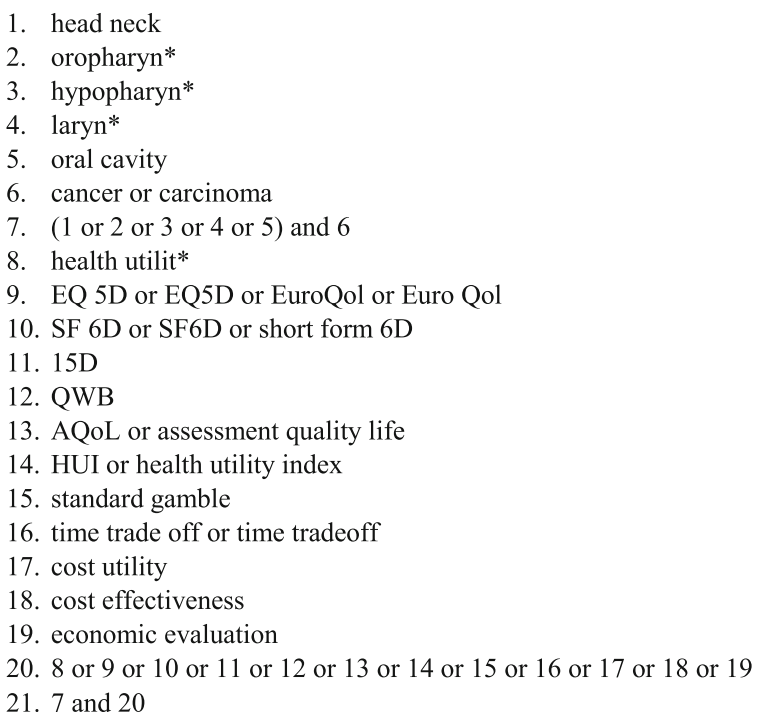

Fig. 1 Free-text terms for electronic database searching coverage of HSUVs [25, 26], we identified a few relevant free-text terms by referring to the published recommendations [26] and recent analogous systematic reviews [25, 27, 28]. Tool- (e.g. EQ-5D) and method-specific (e.g. SG) terms were combined with vocabulary related to HNC including the most frequent cancer sites; in using free-text terms, we considered that some instruments may be referred to or spelled in different ways. We did not explicitly included VAS among the keywords, due to the above-mentioned limitations in using this tool for measuring utility. Other search strings were used to identify cost-effectiveness and cost-utility studies using HSUVs to calculate QALYs. We searched directly utility weights in the Tufts Cost-Effectiveness Analysis (CEA) Registry [29] and the University Sheffield School of Health and Related Research Health Utilities Database (ScHARRHUD) [30]. An additional search was carried out of the Health Economics Research Centre (HERC) database [31] to retrieve mapping studies deriving utility values from non-preference based instruments in HNC. We selected the relevant databases based on previous recommendations [26] and systematic reviews on the topic [32]. Web searches of grey literature were not performed to avoid obtaining contents which are frequently subject to changes and cannot be identified in a systematic manner.

All search results were extracted in an Excel spreadsheet and duplicates removed. Titles and abstracts were screened by two independent reviewers and records excluded if not meeting the inclusion criteria; full-text papers were retrieved in case of doubtful results. Articles estimating $\mathrm{HNC}$ utility values using established methods were included; studies using the VAS instrument were not considered for inclusion, unless alongside other valuation techniques. This choice is consistent with the suggestion that VAS should be used as an introductory task but not as a definitive method to elicit utility values alone [33]. The included studies had to be published as full-text with no time or language restrictions; conference abstracts, editorials, and reviews were not suitable for inclusion. Studies were excluded if they did not report original utility values in $\mathrm{HNC}$; however, the bibliography of studies referring to secondary sources for HSUVs was checked to avoid missing any relevant publications. The authors resolved any disagreements by discussion until consensus was reached.

The characteristics of the included studies were extracted by the first reviewer (MM) using a form developed following previous studies [25-27], and subsequently crosschecked by the other author (JC). Information collected included: study country, study design, sample size, valuation technique, administration method, cancer subsite addressed, and clinical and demographic characteristics of respondents. For each 
HSUV, we recorded the number of respondents, the point estimate (i.e. mean or median) and its measure of variance (e.g. standard deviation); the same information was collected for each study subgroup (or time point) whenever applicable.

Although there are no agreed reporting standards for HSUVs studies, the methodological quality of each included study was evaluated through a set of generic criteria as reported by the guidelines from Papaioannou et al. [26]. Thereafter, one point was awarded to each of the following criteria: (1) sample size $\geq 100$; (2) description of respondent selection and recruitment; (3) description of inclusion/exclusion criteria; (4) response rate $\geq 60 \%$ [34]; (5) reporting of the amount and reasons of loss to follow-up (only for longitudinal studies); (6) reporting of missing data pattern and methods to deal with it; (7) appropriateness of measure (based on the authors' judgment). Lastly, the scores were summed for each article to yield an overall quality score, ranging from 0 to 7 where higher scores indicated higher quality [35]. Any other problems arising from the studies (criterion 8) were narratively discussed. Additionally, the International Society for Pharmacoeconomics and Outcomes Research (ISPOR) recently published a set of recommendations for mapping studies [19] that were used to evaluate the quality of mapping studies retrieved by the systematic search.

\section{Results}

\section{Study selection}

The PRISMA diagram [24] for this literature search is presented in Fig. 2. In total, the search strategy identified 1048 articles: 1046 were retrieved by searching the online databases (PubMed; EMBASE; The Cochrane Library; CEA Registry; HERC database), and two by manually searching the bibliography of model-based economic evaluations retrieved from the online search. No articles were obtained from the ScHARRHUD database. After removing 743 duplicates, 305 records were scanned for title/abstract and 221 were excluded in this first phase for a variety of reasons reported in the chart. Subsequently, 84 full-text articles were retrieved and a further 56 records were excluded for not complying with the inclusion/exclusion criteria. Accordingly, 28 studies were definitively included in the review.

\section{Study characteristics}

The 28 journal articles included in the review are categorized into three groups: studies using direct elicitation methods $(n=10)$, studies administering MAUIs $(n=13)$ and studies deriving HSUVs using mapping $(n=3)$; two studies $[36,37]$ adopted both direct methods and MAUIs. The characteristics of the 25 studies using direct and indirect techniques (i.e. MAUIs) are listed in Table 1, while

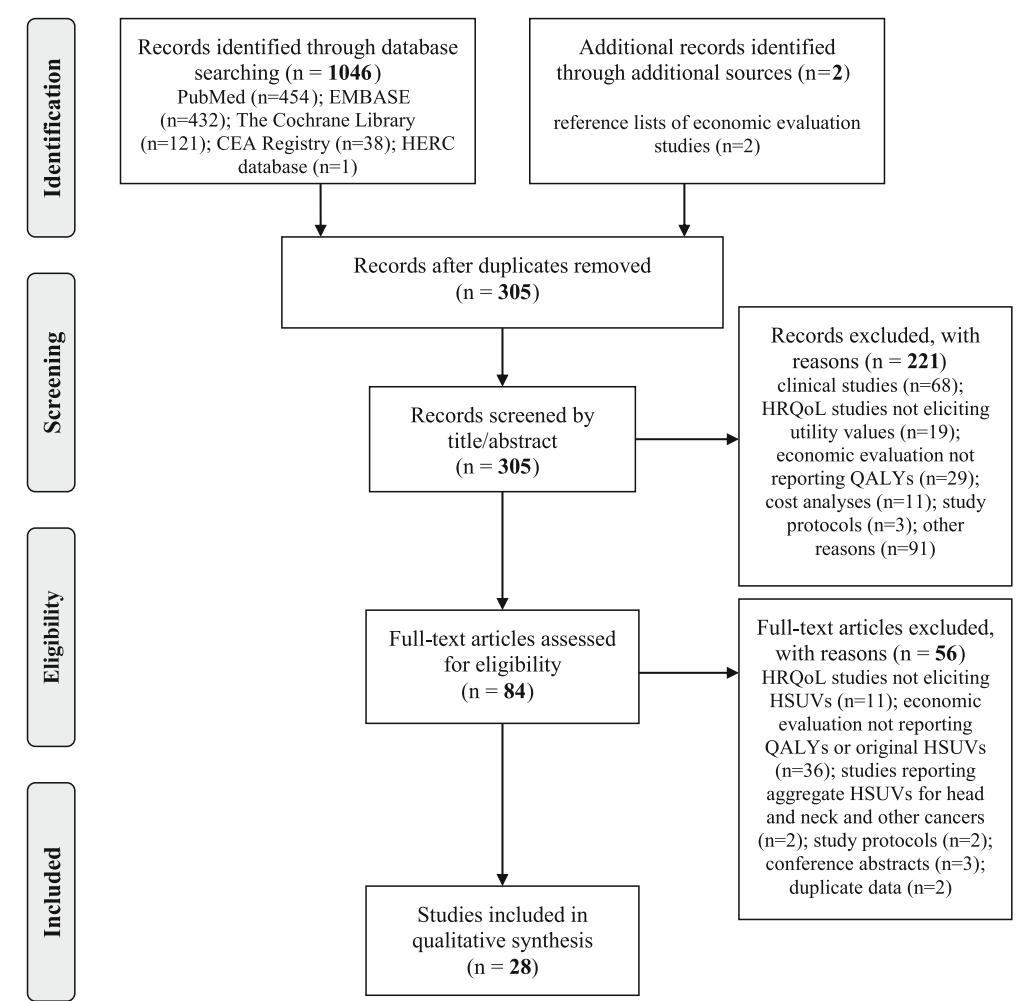

Fig. 2 PRISMA flow chart 
Table 1 Studies estimating HSUVs in HNC using direct or indirect methods ( $n=25$ )

\begin{tabular}{|c|c|c|c|c|c|c|c|c|c|}
\hline $\begin{array}{l}\begin{array}{l}\text { Author } \\
\text { (year) }\end{array} \\
\end{array}$ & Country & Study design & $\begin{array}{l}\text { Cancer } \\
\text { subsite(s) }\end{array}$ & $\begin{array}{l}\text { Valuation } \\
\text { method }\end{array}$ & $\begin{array}{l}\text { Mode of } \\
\text { administration }\end{array}$ & Sample size & Response rate & Participants & $\begin{array}{l}\text { (Mean) age; } \\
\% \text { male }\end{array}$ \\
\hline $\begin{array}{l}\text { Aro } \\
\text { (2016) [56] }\end{array}$ & Finland & Longitudinal & All & $15 \mathrm{D}$ & $\begin{array}{l}\text { Self- } \\
\text { completion } \\
\text { (by post) }\end{array}$ & 214 & $72 \%$ & Patients & $63.0 ; 66 \%$ \\
\hline $\begin{array}{l}\text { Conway } \\
\text { (2012) [38] }\end{array}$ & Australia & Cross-sectional & Oropharynx & SG & $\begin{array}{l}\text { 1-h group } \\
\text { session }\end{array}$ & 99 & $84 \%$ & Healthy subjects & $43.0 ; 54 \%$ \\
\hline $\begin{array}{l}\text { de Almeida } \\
\text { (2014) [44] }\end{array}$ & US & Cross-sectional & Oropharynx & SG; VAS & $\begin{array}{l}\text { Face-to-face } \\
\text { interview }\end{array}$ & 59 & NA & $\begin{array}{l}\text { Healthy subjects } \\
(n=50) \text { and } \\
\text { experts }(n=9)\end{array}$ & $\begin{array}{l}\text { Healthy } \\
\text { subjects: } \\
\text { 34.8; } 42 \% \text {. } \\
\text { Experts: } \\
\text { 45.3; 89\% }\end{array}$ \\
\hline $\begin{array}{l}\text { del Barco } \\
\text { Morillo } \\
\text { (2016) [47] }\end{array}$ & Spain & Longitudinal & All & EQ-5D-3 L & NA & 40 & NA & Patients & $\begin{array}{l}61 \text { (Median); } \\
87 \%\end{array}$ \\
\hline $\begin{array}{l}\text { Govers } \\
\text { (2016) [54] }\end{array}$ & Netherlands & Cross-sectional & $\begin{array}{l}\text { Oral } \\
\text { cavity }\end{array}$ & EQ-5D-3 L & $\begin{array}{l}\text { Self- } \\
\text { completion } \\
\text { (by post) }\end{array}$ & 181 & $62 \%$ & Patients & $64.4 ; \geq 50 \%$ \\
\hline $\begin{array}{l}\text { Hamilton } \\
\text { (2016) [39] }\end{array}$ & UK & Cross-sectional & Larynx & ТTO & $\begin{array}{l}\text { Face-to-face } \\
\text { interview }\end{array}$ & 114 & NA & $\begin{array}{l}\text { Healthy subjects } \\
(n=51) \text { and COPD } \\
\text { patients }(n=63)\end{array}$ & $67.3 ; 49 \%$ \\
\hline $\begin{array}{l}\text { Higgins } \\
\text { (2011) [58] }\end{array}$ & Canada & Cross-sectional & Larynx & HUI3 & Self-completion & 30 & NA & Patients & NA \\
\hline $\begin{array}{l}\text { Hollenbeak } \\
\text { (2001) [40] }\end{array}$ & US & Cross-sectional & All & тTO & NA & 8 & $80 \%$ & Patients & NA \\
\hline $\begin{array}{l}\text { Jalukar } \\
\text { (1998) [41] }\end{array}$ & US & Cross-sectional & All & ТTO & $\begin{array}{l}\text { Self- } \\
\text { completion } \\
\text { (on site for } \\
\text { patients; by } \\
\text { email for } \\
\text { healthcare } \\
\text { professionals) }\end{array}$ & 185 & $\begin{array}{l}\text { Patients: 78\%; } \\
\text { healthcare } \\
\text { professionals: } \\
\text { 42\%; } \\
\text { students: NA }\end{array}$ & $\begin{array}{l}\text { Patients }(n=49) ; \\
\text { healthcare } \\
\text { professionals } \\
(n=50) ; s \\
\text { tudents }(n=86)\end{array}$ & $\begin{array}{l}\text { Patients: } 57.2 ; \\
71 \% \\
\text { Healthcare } \\
\text { professionals: } \\
\text { 40.1; } \\
\text { 40\%; students: } \\
\text { NA }\end{array}$ \\
\hline $\begin{array}{l}\text { Kent } \\
\text { (2015) [59] }\end{array}$ & US & Cross-sectional & $\begin{array}{l}\text { Oral } \\
\text { cavity } \\
\text { and pharynx }\end{array}$ & $\begin{array}{l}\text { SF-6D/ } \\
\text { VR-6D }\end{array}$ & $\begin{array}{l}\text { Mail or } \\
\text { telephone }\end{array}$ & 580 & $62 \%$ & Patients & $67.7 ; 60 \%$ \\
\hline $\begin{array}{l}\text { Llewellyn- } \\
\text { Thomas } \\
\text { (1993) [45] }\end{array}$ & Canada & Longitudinal & Larynx & TTONAS & Interview & 66 & NA & Patients & $N A ; 86 \%$ \\
\hline $\begin{array}{l}\text { Loimu } \\
\text { (2015) [57] }\end{array}$ & Finland & Longitudinal & $\begin{array}{l}\text { Pharynx, } \\
\text { larynx, } \\
\text { nasal cavity }\end{array}$ & $15 \mathrm{D}$ & $\begin{array}{l}\text { Self- } \\
\text { completion: } \\
\text { on site (first } \\
\text { assessment); } \\
\text { by post } \\
\text { (afterwards) }\end{array}$ & 64 & $76 \%$ & Patients & $61.6 ; 75 \%$ \\
\hline $\begin{array}{l}\text { Marcellusi } \\
\text { (2015) [36] }\end{array}$ & Italy & Cross-sectional & All & $\begin{array}{l}\text { TTO; EQ- } \\
\text { 5D-3 L }\end{array}$ & Computer-guided & 79 & NA & Patients & $65.0 ; 78.5 \%$ \\
\hline $\begin{array}{l}\text { Noel } \\
\text { (2015) [37] }\end{array}$ & Canada & Cross-sectional & All & $\begin{array}{l}\text { SG; TTO; } \\
\text { VAS; EQ- } \\
\text { 5D-5 L; } \\
\text { HUI3 }\end{array}$ & $\begin{array}{l}\text { Face-to-face } \\
\text { interview }\end{array}$ & 100 & $79 \%$ & Patients & $61.0 ; 75 \%$ \\
\hline $\begin{array}{l}\text { Ouattassi } \\
\text { (2016) [48] }\end{array}$ & Morocco & Cross-sectional & All & EQ-5D-3 L & $\begin{array}{l}\text { Self- } \\
\text { completion }\end{array}$ & 120 & NA & Patients & $57.0 ; 60 \%$ \\
\hline $\begin{array}{l}\text { Parrilla } \\
\text { (2015) [49] }\end{array}$ & Italy & Longitudinal & Larynx & EQ-5D & $\begin{array}{l}\text { Self- } \\
\text { completion }\end{array}$ & 30 & NA & Patients & $68.7 ; 93 \%$ \\
\hline $\begin{array}{l}\text { Pickard } \\
\text { (2016) [52] }\end{array}$ & US & Cross-sectional & All & EQ-5D-3 L & $\begin{array}{l}\text { Self- } \\
\text { completion }\end{array}$ & 50 & NA & Patients & 56.0; NA \\
\hline $\begin{array}{l}\text { Pottel } \\
\text { (2015) [55] }\end{array}$ & Belgium & Longitudinal & All & EQ-5D-3 L & $\begin{array}{l}\text { Self- } \\
\text { completion } \\
\text { or interview } \\
\text { on site (first } \\
\text { assessment); } \\
\text { by post (afterwards) }\end{array}$ & 81 & $81 \%$ & Patients & $72.0 ; 86 \%$ \\
\hline
\end{tabular}


Table 1 Studies estimating HSUVs in HNC using direct or indirect methods ( $n=25)$ (Continued)

\begin{tabular}{|c|c|c|c|c|c|c|c|c|c|}
\hline $\begin{array}{l}\text { Ramaekers } \\
(2011) \text { [51] }\end{array}$ & Netherlands & Cross-sectional & All & EQ-5D-3 L & Self-completion & 396 & $93 \%$ & Patients & $63.2 ; 70 \%$ \\
\hline $\begin{array}{l}\text { Ringash } \\
(2000) \text { [42] }\end{array}$ & Canada & Cross-sectional & Larynx & TTO & $\begin{array}{l}\text { Face-to-face } \\
\text { interview }\end{array}$ & 120 & $49 \%$ & Patients & $65 ; 83 \%$ \\
\hline $\begin{array}{l}\text { Rogers } \\
(2006) \text { [50] }\end{array}$ & UK & Cross-sectional & $\begin{array}{l}\text { Oral cavity } \\
\text { and } \\
\text { oropharynx }\end{array}$ & EQ-5D-3 L & $\begin{array}{l}\text { Self-completion } \\
\text { (by post) }\end{array}$ & 224 & $64 \%$ & Patients & $65 ; 58 \%$ \\
\hline $\begin{array}{l}\text { Szabo } \\
(2012) \text { [20] }\end{array}$ & Canada & Cross-sectional & $\begin{array}{l}\text { Larynx, lip, } \\
\text { oral cavity, } \\
\text { oropharynx }\end{array}$ & SG & $\begin{array}{l}\text { Interview using } \\
\text { script and prop }\end{array}$ & 101 & $95 \%$ & Healthy subjects & $47 ; 48 \%$ \\
\hline $\begin{array}{l}\text { Truong } \\
\text { (2016) [53] }\end{array}$ & US & $\mathrm{RCT}$ & $\begin{array}{l}\text { Oropharynx, } \\
\text { hypopharynx, } \\
\text { larynx }\end{array}$ & EQ-5D-3 L & Self-completion & 818 & $87 \%$ & Patients & $\begin{array}{l}\text { Arm CIS: } 56.1 \text {; } \\
86 \% \text {. } \\
\text { Arm CET/CIS: } \\
\text { 57.3; } 89 \%\end{array}$ \\
\hline $\begin{array}{l}\text { van der } \\
\text { Donk } \\
\text { (1995) [46] }\end{array}$ & Netherlands & Cross-sectional & Larynx & $\begin{array}{l}\text { TTO/SG/ } \\
\text { VAS }\end{array}$ & $\begin{array}{l}\text { Face-to-face } \\
\text { interview }\end{array}$ & 39 & NA & $\begin{array}{l}\text { Laryngeal cancer } \\
\text { patients }(n=10), \\
\text { FOM cancer } \\
\text { patients }(n=10), \\
\text { experts }(n=9), \\
\text { healthy subjects } \\
(n=10)\end{array}$ & $\begin{array}{l}\text { Laryngeal } \\
\text { cancer } \\
\text { patients: 62; } \\
\text { FOM cancer } \\
\text { patients: 56; } \\
\text { experts: 43; } \\
\text { healthy } \\
\text { subjects: } 36\end{array}$ \\
\hline $\begin{array}{l}\text { Weiss } \\
\text { (1994) [43] }\end{array}$ & US & Cross-sectional & $\begin{array}{l}\text { Pharynx, } \\
\text { larynx }\end{array}$ & TTO & NA & 3 & NA & Clinical experts & NA \\
\hline
\end{tabular}

Abbreviations: CIS radiation-cisplatin without cetuximab, CET/CIS radiation-cisplatin with cetuximab, COPD chronic obstructive pulmonary disease, EQ-5D-3 L EuroQol 5-dimension 3-Level, EQ-5D-5 L EuroQol 5-dimension 5-Level, FOM floor-of-the-mouth, HNC head and neck cancer, HSUV health state utility value, HUI3 Health Utility Index Mark 3, NA not available, SF-6D Short Form-6-dimension, SG standard gamble, $T T O$ time trade off, VAS visual analogue scale, $V R-6 D$ Veterans RAND-6-dimension

the three mapping studies are separately described in Table 2 .

\section{Studies using direct or indirect methods}

Among the studies using direct elicitation techniques, SG alone was adopted in two cases [20, 38] and TTO alone in five [39-43]. In four studies [37, 44-46], more than one direct methodology (i.e. SG, TTO, VAS) was adopted to derive utility values. The study by Noel et al. [37] compared these direct techniques with MAUIs (i.e. EQ-5D, HUI3), while a further study [36] used both TTO and EQ-5D instruments.
In studies administering MAUIs, EQ-5D was the most common $(n=11)$; five of these studies [36, 37, 47-49] did not report which scoring algorithm was used, two studies $[50,51]$ explicitly adopted the UK algorithm, another two $[52,53]$ adopted the US one, one study [54] used the Dutch tariff and another one [55] the Belgian one. Moreover, nine of the studies using EQ-5D [36, 47, 48, 50-55] explicitly referred to the 3-level version (EQ-5D-3 L) and one [37] to the newer 5-level one (EQ-5D-5 L); one study [49] did not specify the instrument's version adopted. Additional generic, preference-based HRQoL tools retrieved by our search were 15D $(n=2)$, HUI3 $(n=2)$ and

Table 2 Mapping studies predicting HSUVs in HNC $(n=3)$

\begin{tabular}{|c|c|c|c|c|c|c|c|c|c|c|}
\hline $\begin{array}{l}\text { Author } \\
\text { (year) }\end{array}$ & Country & $\begin{array}{l}\text { Mapping } \\
\text { technique }\end{array}$ & $\begin{array}{l}\text { From } \\
\text { (tool 1) }\end{array}$ & $\begin{array}{l}\text { To } \\
\text { (tool 2) }\end{array}$ & $\begin{array}{l}\text { Sample's } \\
\text { description } \\
\text { (algorithm) }\end{array}$ & $\begin{array}{l}\text { Sample size } \\
\text { (algorithm) }\end{array}$ & $\begin{array}{l}\text { Study ref. } \\
\text { (algorithm) }\end{array}$ & $\begin{array}{l}\text { Sample's description } \\
\text { (tool 1) }\end{array}$ & $\begin{array}{l}\text { Sample size } \\
\text { (tool 1) }\end{array}$ & $\begin{array}{l}\text { Study ref. } \\
\text { (tool 1) }\end{array}$ \\
\hline Chan (2014) [60] & Canada & OLS & UW QOL v4 & EQ-5D-3 L & $\begin{array}{l}\text { Patients } \\
\text { treated } \\
\text { for HNC }\end{array}$ & $\begin{array}{l}89 \text { (estimation); } \\
48 \text { (validation) }\end{array}$ & & & & \\
\hline Parthan (2009) [61] & UK & $\begin{array}{l}\text { Published } \\
\text { algorithm }\end{array}$ & $\begin{array}{l}\text { EORTC QLQ- } \\
\text { C30 }\end{array}$ & EQ-5D-3 L & $\begin{array}{l}\text { Patients } \\
\text { with liver } \\
\text { metastases }\end{array}$ & 75 & $\begin{array}{l}\text { Krabbe } \\
\text { (2004) [63] }\end{array}$ & $\begin{array}{l}\text { Patients with } \\
\text { locally advanced } \\
\text { inoperable HNC }\end{array}$ & 358 & $\begin{array}{l}\text { Vermorken } \\
\text { (2007) [66] }\end{array}$ \\
\hline Yong (2012) [62] & Canada & $\begin{array}{l}\text { Published } \\
\text { algorithm }\end{array}$ & SF-36 & HUI2 & $\begin{array}{l}\text { Various } \\
\text { patients }\end{array}$ & 6921 & $\begin{array}{l}\text { Nichol } \\
(2001) \text { [64] }\end{array}$ & $\begin{array}{l}\text { Patients with } \\
\text { early stage } \\
\text { nasopharyngeal } \\
\text { cancer }\end{array}$ & 51 & $\begin{array}{l}\text { Pow } \\
(2006) \text { [65] }\end{array}$ \\
\hline
\end{tabular}


SF-6D $(n=1)$; no studies used the QWB scale or the AQoL-8D utility instrument.

The 25 articles reported on HNC utility-related studies conducted in several European (Belgium, $n=1$; Finland, $n=2$; Italy, $n=2$; Netherlands, $n=3$ Spain; $n=1$ United Kingdom, $n=2$ ) and non-European countries (Australia, $n=1$; Canada, $n=5$ Morocco, $n=1$; United States, $n=7$ ). The great majority of the HSUVs came from cross-sectional surveys $(n=18)$; the remaining articles $(n=7)$ adopted a longitudinal design, including five prospective cohort studies $[45,49,55-57]$ and two clinical trials $[47,53]$.

Sample sizes varied widely from 3 [43] to 818 [53], with a mean of 152 respondents per study. The response rate was between 49\% [42] and 95\% [20]. In most of the studies $(n=18)$, the participants were HNC patients at various stages of disease and treatment pathway; in two studies $[20,38]$ healthy individuals from the general population were surveyed through the SG techniques, while in one case [43] the utility assessment was based on a consultation with a panel of experienced physicians. The remaining four studies $[39,41,44,46]$ retrieved utility measures from multiple subjects (i.e. healthy people, clinical experts, HNC patients and patients with other medical conditions) and reported HSUVs from each group separately.

In studies recruiting $\mathrm{HNC}$ patients, most were male and the mean age was always above 55 . Conversely, responders were generally younger and with a higher proportion of females in studies surveying individuals from the general population or clinical experts. The range of cancer subsites addressed by each study was quite broad: ten studies [36, 37, 40, 41, 47, 48, 51, 52, 55, 56] generally investigated utility in $\mathrm{HNC}$ without specifying any cancer site, six $[39,42,45,46,49,58]$ were related to laryngeal cancer, two $[38,44]$ addressed cancer in the oropharynx, one [54] recruited patients affected by cancer in the oral cavity and the remaining six [20, 43, $50,53,57,59]$ focused on selected multiple sites (e.g. oropharynx, hypopharynx, and larynx).

The most common way $(n=12[41,48-58])$ of collecting utility data was by self-completion of a written survey (administered on site or by post/e-mail), followed by faceto-face interviews ( $n=6[37,39,42,44-46])$; four studies adopted different administration options including group session $(n=1$ [38]), telephone or mail interview $(n=1$ [59]), interview using a script/prop $(n=1$ [20]), and computer-guided data collection $(n=1$ [36]). The administration method was not specified in three cases [40, 43, 47]. When HSUVs were obtained from the patients, the survey (or the interview) was usually scheduled during a clinical appointment or a hospital admission; in longitudinal studies [55, 57], surveys after the first were frequently delivered by post to the patient's home address.

\section{Mapping studies}

The three studies deriving HSUVs in HNC using a mapping technique are described in Table 2. Among them, only one [60] developed an original mapping algorithm using responses from HNC patients and was retrieved from the HERC database. Ordinary Least Square (OLS) regression was applied to establish a statistical relationship between the University of Washington Quality of Life questionnaire version 4 (UW QOL v4) and the EQ5D-3 L using a dataset of 89 patients treated for HNC. Thereafter, the responses of an additional 48 patients enrolled in the study were used as a validation database.

The other two studies [61, 62] were model-based economic evaluations reporting HSUVs for several HNC-related health states by applying previously published algorithms $[63,64]$ to $\mathrm{HRQoL}$ data retrieved from the literature $[65,66]$.

\section{Overview of HSUVs}

A total of 346 original HSUVs were retrieved from 27 studies included in the review (Additional file 1: Table S1), since one study [41] reported results only graphically in the article. The studies $[37,44,46]$ providing the highest number of HSUVs (i.e. over 40) either adopted multiple techniques or interviewed several groups of respondents that yielded different values for each health state. In other cases [45, 49, 53, 55-57, 62], different HSUVs have been collected by the same participants over the study time points. HSUVs were reported as means in the great majority of studies $(n=25)$, of which four $[20,38,51,53]$ also reported the median; the remaining two studies $[47,55]$ calculated a median value only. Among the measures of variance, standard deviation was the most frequently adopted $(n=12)$, followed by the min-max range $(n=7)$, and the interquartile range $(n=5)$; several studies reported more than one measure type. In some cases $[39,43,46$, $58,62]$, no measures of variability were reported, thus limiting the usefulness of the utility data.

\section{Study quality assessment}

The quality assessment of the 25 studies using direct or indirect methods was based upon eight criteria, of which seven were given a score (Table 3). In all studies, the instrument adopted to estimate HSUVs was considered appropriate in relation with the participants enrolled. Additionally, most studies (84\%) reported a description of the participants recruitment process, whilst only $56 \%$ of them clearly stated the inclusion/exclusion criteria. Information on missing data and techniques to deal with them were reported by a limited number of studies (24\%). In half of the studies, the sample size was rather small $(<100)$ and response rate was either low $(<60 \%)$ or not reported. In reviewing these studies, we highlighted a few additional issues (criterion 8 [26]) that should be 


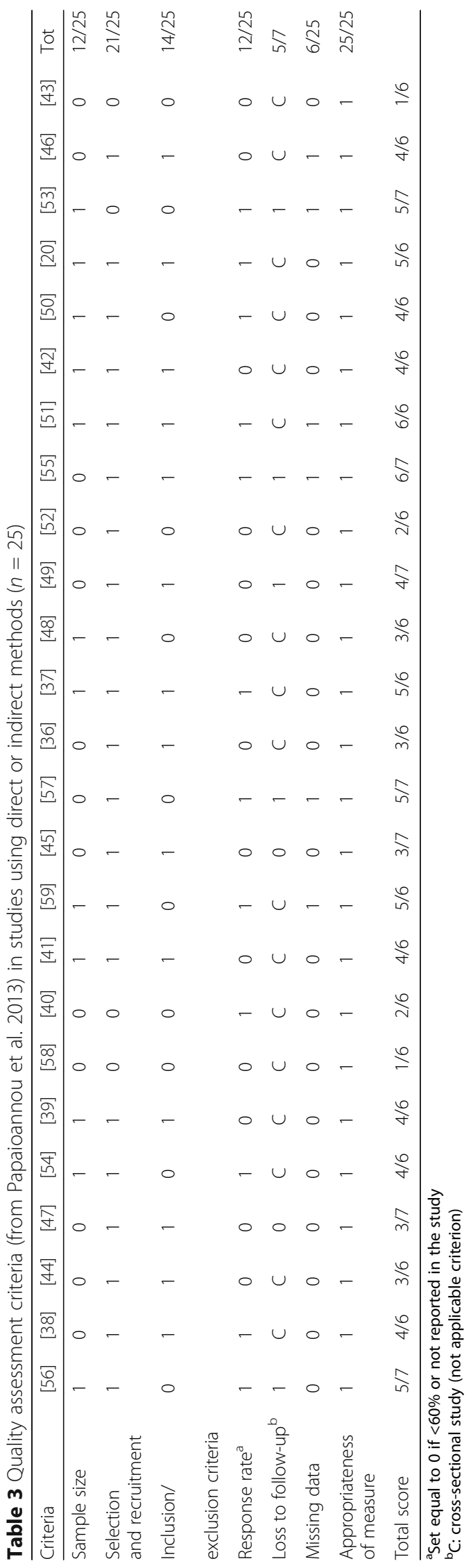


considered when selecting sources to populate health economic models with utility parameters. First, some of the included studies are quite dated (published before 2000), thus describing health states that might not be realistic nowadays because of emerging treatment modalities, improvements in treatment-related morbidity and organ preservation techniques. Second, there might be potential sources of bias in reporting HRQoL results in clinical studies investigating one or more interventions, although the number of comparative trials retrieved by our search was very limited. Third, in some studies [36, 48, 59, 60], and especially those analyzing HRQoL in multiple cancers including head and neck $[36,59]$, patient's characteristics (e.g. cancer stage/site, treatment phase) are poorly reported, thus making it difficult to match the study's HSUVs with the health states described in a cost-effectiveness model. Lastly, the great majority of studies are cross-sectional surveys, representing the quickest and cheapest method for gathering HRQoL data; however, longitudinal data collections are often more valuable since they facilitate capture of changes in utility values as cancer progresses through different phases.

With reference to mapping, we retrieved only one original algorithm [60] through the search, thus preventing a comparative evaluation of studies. This study presented a four-variable model to predict EQ-5D-3 L utilities using OLS regression; coefficient values and error terms were clearly reported and box-plot distributions of actual and predicted utilities provided. However, the authors did not justify the model choice in relation to the observed EQ-5D distribution, nor any additional tests or judgments made. The goodness-of-fit was presented as R,2 mean absolute error (MAE) and root mean squared error (RMSE), which are considered of limited value in the mapping field. No demographic or clinical variables were included as covariates, which was recognized as a study limitation by the same authors. Moreover, when the sample size is small (as it was in this study), the most recent guidelines do not recommend splitting it for empirical validation [19].

\section{Discussion}

This study reviews systematically published studies reporting HSUVs in HNC. Compared to a previous review [23], many more studies have been identified, most of which use the EQ-5D and were published from 2011 onwards. Overall, this review shows that HNC patients suffer from substantial HRQoL impairment over the different disease phases. However, there is a lack of research into the HRQoL in the recurrent and/or metastatic health states, with only one study [47] reporting a median EQ-5D utility value (i.e. 0.7) from the patients, which is less useful for the purposes of economic evaluation that focuses on mean costs and effects. Another study [44] elicits values for a range of recurrent disease states from healthy subjects and clinical experts using SG and VAS and obtains extremely heterogeneous results across the types of participants and methods. The same paucity of HSUVs was observed for treatment-related complications, which are addressed by three studies $[20,44,45]$ only, possibly because of the infrequency of some of these events that restricts the data from patients in that health state.

Differences in utilities were found across studies even in the pre-treatment state. The choice of baseline utility is particularly relevant because it affects the incremental gain achievable by different therapeutic options [13], thus potentially biasing the estimated cost-effectiveness. The two Finnish studies $[56,57]$ using the 15D yielded higher utility values in patients shortly after diagnosis than those using the EQ-5D [53, 54]. This phenomenon has previously been observed in studies addressing other medical conditions $[32,67,68]$. There are many possible explanations for these discrepancies: different number of dimensions, the EQ-5D has generally been valued using TTO rather than VAS [69], the preference weights have come from different populations (a Finnish value set is usually adopted for 15D) [67], and the EQ-5D, unlike the $15 \mathrm{D}$, can take negative values $[69,70]$. The participants' characteristics might have also affected study results. For example, a study [55] addressing HRQoL in patients aged $\geq 65$ years with HNC consistently provided lower HSUVs than other studies in either the pretreatment, treatment, and follow-up phases, probably because of comorbidities and functional impairments usually affecting elderly people independently from cancer. Moreover, the use of different scoring algorithms may have contributed to variation in HSUVs in studies administering the EQ-5D.

Heterogeneity in utility values was particularly evident in the studies applying more than one technique to evaluate the same health state. Among them, in a study reporting HSUVs for different treatments, treatmentrelated complications, and remission/recurrence states in oropharyngeal cancer [44], the values obtained using a VAS scale were consistently lower that for the SG. In the study by Marcellusi et al. [36], patients in follow-up after treatment for HNC reported lower utility values when performing the TTO task than when responding to the EQ-5D questionnaire. Another study [37] compared five different (direct and indirect) methods to retrieve HSUVs from patients experiencing a similar health state (i.e. three months after completion of treatments and no evidence of recurrent disease). Unlike Marcellusi et al. [36], the method yielding the highest utility value in the overall sample $(n=100)$ was TTO (0.94), followed by SG (0.91), EQ-5D (0.82), VAS (0.76) 
and HUI3 (0.75). That VAS scores are consistently lower than SG scores is well-known in the literature; in 2001, Torrance et al. [33], after reviewing several studies, concluded that the relationship between the two instruments can be represented by a concave curve passing through 0 and 1 . Moreover, the indirect methods involving MAUIs have been shown to yield systematically lower utility values than the direct ones in a wide range of diseases [71] for a variety of reasons. First, in MAUIs participants are not asked to consider their health status relative to death and thus, there is no disincentive in reporting more severe health problems [72]. Second, respondents are forced to describe their complex medical conditions through a limited number of attributes, thus ignoring any positive feelings that would boost utility values. Third, it is likely that the general population used to obtain tariffs for MAUIs make a different trade-off between a given health state and death because they tend to be younger and healthier. Finally, the vignettes described in direct valuation tasks are usually more detailed than the MAUI health states [71]. In studies comparing alternative MAUIs, EQ-5D has been shown to provide higher utilities values compared to HUI2 and HUI3, which in turn yield higher values than SF-6D. As for the differences between EQ-5D and 15D, potential explanations are likely to be found in descriptive systems, preference measurement, source of community preferences, and scoring methods [73].

In addition, studies can be classified by the type of responders who valued the health states, either patients or healthy subjects. In the literature, some argue that patients are best placed to value the relevant health states, while others advocate valuation by healthy people who will not directly benefit from a new treatment but, in tax-based systems, will bear its cost. The latter claim that this will provide an unbiased estimate of the hypothetical health states $[7,74]$ and more consistency across appraisal of very different interventions. The review by Komatsuzaki et al. [23] showed that patients usually reported lower utilities than physicians and healthy people for health states associated to HNC. In this review, only a few studies recruited participants from the general population, thus limiting the number of utilities comparisons across different types of responders. One study [46] confirms the conclusions reached by the previous review [23], whilst others [41, 44] found healthy subjects consistently providing lower utility estimates compared to patients and healthcare professionals.

This study facilitates the identification of HSUVs for use in future HNC economic evaluations. The number of retrieved studies was quite large, with almost 350 distinct HSUVs collected from them. Most of the utility values were collected during the treatment phase or shortly after the completion of treatment, whilst limited evidence is available for the health-related utility assessment in HNC recurrent and end-of-life states. Due to the variety of health state definitions and valuation techniques across the studies, we were not able to perform a quantitative synthesis of the results [3]. Moreover, unlike cost-effectiveness studies where structured guidelines exist to support authors and reviewers in assessing their quality $[75,76]$, recommendations for valuation studies specifically aimed at measuring HSUVs are more fragmented or method-specific [10]. In this review, the assessment of study quality was based on a set of generic recommendations elaborated by a previous study [26] and arbitrarily modified to allow a quantitative scoring of the studies adopting direct and indirect techniques to estimate HSUVs; for mapping studies, we relied instead on recent ISPOR guidelines [19].

Although there is no universally accepted theoretical basis for choosing direct or indirect methods [71], the use of the EQ-5D, is favored by several agencies including NICE, the Canadian Agency for Drugs and Technologies in Health and the French National Authority for Health [3]. In a recent position statement [77], NICE recommends the use of EQ-5D-3 L for base-case analyses, or mapping EQ-5D-5 L responses onto the $3 \mathrm{~L}$ valuation set, to derive HSUVs, since further research is needed to explore the impact of adopting the EQ-5D$5 \mathrm{~L}$ valuation set on technology appraisal. In modelbased cost-effectiveness studies, where there is a choice of HSUVs, those using the value set of the jurisdiction for which a decision is being made are usually preferred. Moreover, HSUVs should be collected from studies enrolling patients with demographic and clinical characteristics that mostly resemble those of potential recipients of the intervention under investigation in the model. Until now, studies relying on direct techniques represent the only available source to retrieve HSUVs for recurrent disease, palliative states, or treatment-related complications in HNC. Although considered as qualitatively inferior to MAUIs [3], these methods can provide values for cost-effectiveness analyses where the 'vignettes' presented in the choice task fit with the health states addressed in the model. Finally, in the absence of preference-based data, mapping from disease-specific instruments to generic MAUIs may represent a valuable alternative [74]; however, the only algorithm published to date in HNC [60] does not map from one of the HRQoL tools most frequently adopted in cancer studies, such as the European Organization for Research and Treatment of Cancer 30-item Quality of Life Core Questionnaire (EORTC QLQ-C30 [78]) and the Functional Assessment of Cancer Therapy - General (FACT-G [79]). Greater availability of mapping functions would facilitate the comparison of treatments using HRQoL data from many randomized controlled trials 
that only collected disease-specific health status information. Overall, the use of different techniques for utility elicitation might have substantial implications in cost-utility analyses; for example, it has been shown [71] that MAUIs, compared to direct valuation, tend to favor non-lifesaving treatments over interventions preventing or delaying death. Thus, regulatory bodies should avoid a mixture of methods in their decision processes to avoid a biased allocation of healthcare resources. Moreover, health economic modelers are always recommended to extensively test the uncertainty around the utility parameters in sensitivity analyses [71].

\section{Conclusions}

This study improves understanding of preference-based HRQoL measurement in HNC by systematically reviewing and critically evaluating studies that estimated HSUVs in this cancer setting. Utility values are an essential parameter but also a major source of uncertainty in model-based economic evaluations, where it is common to select them from a single study based on clinical considerations $[3,28]$. Further studies on the health-related utility assessment from HNC patients using MAUIs in recurrent and terminal states are encouraged. Additional research on mapping algorithms to convert diseasespecific HRQoL results onto preference-based HSUVs would be of value in this cancer population. Overall, the methods used to identify utility values within a growing body of HRQoL literature should be increasingly systematic and justified in future studies.

\section{Additional file}

Additional file 1: Table S1. Details of HSUVs reported by the studies $(n=27)$. (DOCX $107 \mathrm{~kb})$

\section{Acknowledgments}

Not applicable.

\section{Funding}

The authors declare that this study received no financial support.

\section{Availability of data and materials}

The datasets supporting the conclusions of this article are included within its tables and additional files.

\section{Authors' contributions}

MM conducted the systematic literature search, extracted, analyzed, and interpreted the data, and drafted the work. JC conceived the work, interpreted the results, and contributed to the manuscript preparation. Both authors read and approved the final version of the manuscript.

\section{Ethics approval and consent to participate}

Not applicable.

\section{Consent for publication}

Not applicable.

\section{Competing interests}

The authors declare they have no competing interests.

\section{Publisher's Note}

Springer Nature remains neutral with regard to jurisdictional claims in published maps and institutional affiliations.

\section{Author details}

${ }^{1}$ Department of Health Services Research and Policy, Faculty of Public Health and Policy, London School of Hygiene and Tropical Medicine, 15-17

Tavistock Place, London WC1H 9SH, UK. ${ }^{2}$ CeRGAS (Research Centre on Health and Social Care Management), Bocconi University, Via Roentgen 1, 20136 Milan, Italy. ${ }^{3} \mathrm{CCBIO}$ (Centre for Cancer Biomarkers), University of Bergen, Postboks 7804, N-5020 Bergen, Norway.

Received: 20 February 2017 Accepted: 24 August 2017

Published online: 02 September 2017

\section{References}

1. Neumann PJ, Goldie SJ, Weinstein MC. Preference-based measures in economic evaluation in health care. Annu Rev Public Health. 2000;21:587-611.

2. Weinstein MC, Torrance G, McGuire A. QALYs: the basics. Value Health. 2009; 12(Suppl 1):S5-9.

3. Wolowacz SE, Briggs A, Belozeroff V, Clarke P, Doward L, Goeree R, et al. Estimating health-state utility for economic models in clinical studies: an ISPOR good research practices task force report. Value Health. 2016;19(6): 704-19.

4. Rashidi AA, Anis AH, Marra CA. Do visual analogue scale (VAS) derived standard gamble (SG) utilities agree with health utilities index utilities? A comparison of patient and community preferences for health status in rheumatoid arthritis patients. Health Qual Life Outcomes. 2006;4:25.

5. Witney AG, Treharne GJ, Tavakoli M, Lyons AC, Vincent $K$, Scott DL, et al. The relationship of medical, demographic and psychosocial factors to direct and indirect health utility instruments in rheumatoid arthritis. Rheumatology. 2006;45(8):975-81

6. Dolan P, Sutton M. Mapping visual analogue scale health state valuations onto standard gamble and time trade-off values. Soc Sci Med. 1997;44(10):1519-30.

7. Whitehead SJ, Ali S. Health outcomes in economic evaluation: the QALY and utilities. Br Med Bull. 2010;96(1):5-21.

8. Post MWM. Definitions of quality of life: what has happened and how to move on. Top Spinal Cord Inj Rehabil. 2014;20(3):167-80.

9. Wiedermann W, Frick U. Using surveys to calculate disability-adjusted lifeyear. Alcohol Res. 2013;35(2):128-33.

10. Attema AE, Edelaar-Peeters $Y$, Versteegh MM, Stolk EA. Time trade-off: one methodology, different methods. Eur J Health Econ. 2013 Jul;14(Suppl 1):S53-64.

11. Richardson J, Khan MA, lezzi A, Maxwell A. Comparing and explaining differences in the magnitude, content, and sensitivity of utilities predicted by the EQ-5D, SF-6D, HUI3, 15D, QWB, and AQoL-8D multi-attribute utility instruments. Med Decis Mak. 2015;35:276-91.

12. Beaudet A, Clegg J, Thuresson PO, Lloyd A, McEwan P. Review of utility values for economic modeling in type 2 diabetes. Value Health. 2014;17(4):462-70.

13. EUnetHTA. Methods for health economic evaluations - a guideline based on current practices in Europe. Copyright (c) EUnetHTA 2014.

14. Hao Y, Wolfram V, Cook J. A structured review of health utility measures and elicitation in advanced/metastatic breast cancer. Clinicoecon Outcomes Res. 2016;8:293-303.

15. Boye KS, Matza LS, Feeny DH, Johnston JA, Bowman L, Jordan JB. Challenges to time trade-off utility assessment methods: when should you consider alternative approaches? Expert Rev Pharmacoecon Outcomes Res. 2014; 14(3):437-50

16. Horsman J, Furlong W, Feeny D, Torrance G. The health utilities index $\left(\mathrm{HUI}^{\oplus}\right)^{\circledR}$ : concepts, measurement properties and applications. Health Qual Life Outcomes. 2003;1:54.

17. Button B. Quality of well-being (QWB) scale. Encyclopedia of quality of life and well-being research; 2014. p. 5314-20. https://doi.org/10.1007/978-94007-0753-5_654.

18. Hawthorne G, Richardson J, Osborne R. The assessment of quality of life (AQoL) instrument: a psychometric measure of health-related quality of life. Qual Life Res. 1999;8(3):209-24.

19. Wailoo AJ, Hernandez-Alava M, Manca A, Mejia A, Ray J, Crawford B, et al. Mapping to estimate health-state utility from non-preference-based outcome measures: an ISPOR good practices for outcomes research task force report. Value Health. 2017;20(1):18-27. 
20. Szabo SM, Dobson RL, Donato BMK, L'Italien G, Hotte SJ, Levy AR. The quality-of-life impact of head and neck cancer: preference values from the Canadian general public. Health Outcomes Res Med. 2012;3(1):e11-23.

21. Hannouf MB, Sehgal C, Cao JQ, Mocanu JD, Winquist E, Zaric GS. Costeffectiveness of adding cetuximab to platinum-based chemotherapy for first line treatment of recurrent or metastatic head and neck cancer. PLoS One. 2012;7(6):e38557. https://doi.org/10.1371/journal.pone.0038557.

22. Liberato NL, Rognoni C, Rubrichi S, Quaglini S, Marchetti M, Gorlia T, et al. Adding docetaxel to cisplatin and fluorouracil in patients with unresectable head and neck cancer: a cost-utility analysis. Ann Oncol. 2012;23(7):1825-32

23. Komatsuzaki Y, Gramegna P, Stephens JM, Botteman MF, Pashos CL, Redaelli A. Preferences and utilities of health outcomes and treatments associated with head and neck cancer: a systematic review. Am J Cancer. 2006;5(1):27-34.

24. Moher D, Shamseer L, Clarke M, Ghersi D, Liberati A, Petticrew M, PRISMA-P Group, et al. Preferred reporting items for systematic review and metaanalysis protocols (PRISMA-P) 2015 statement. Syst Rev. 2015;4:1. https://doi. org/10.1186/2046-4053-4-1.

25. Sampson CJ, Tosh JC, Cheyne CP, Broadbent D, James M. Health state utility values for diabetic retinopathy: protocol for a systematic review and metaanalysis. Systematic review. 2015;4:15. https://doi.org/10.1186/s13643-015-0006-6.

26. Papaioannou D, Brazier J, Paisley S. Systematic searching and selection of health state utility values from the literature. Value Health. 2013;16:686-95.

27. Jeong K, Cairns J. Systematic review of health state utility values for economic evaluation of colorectal cancer. Health Econ Rev. 2016;6(1):36

28. Gheorghe A, Moran G, Duffy H, Roberts T, Pinkney T, Calvert M. Health utility values associated with surgical site infection: a systematic review. Value Health. 2015;18(8):1126-37.

29. CEA Registry. Available at: http://healtheconomics.tuftsmedicalcenter.org/ cear4/Home.aspx. Accessed 18 Nov 2016.

30. ScHARRHUD Health Utilities Database. Available at: http://www.scharrhud. org/. [Accessed 22 Nov 2016].

31. Dakin $\mathrm{H}$. Review of studies mapping from quality of life or clinical measures to EQ-5D: an online database. Health Qual Life Outcomes. 2013;11:151. https://doi.org/10.1186/1477-7525-11-151.

32. Smith-Palmer J, Bae JP, Boye KS, Norrbacka K, Hunt B, Valentine WJ. Evaluating health-related quality of life in type 1 diabetes: a systematic literature review of utilities for adults with type 1 diabetes. Clinicoecon Outcomes Res. 2016;8:559-71.

33. Torrance GW, Feeny D, Furlong W. Visual analog scales: do they have a role in the measurement of preferences for health states? Med Decis Mak. 2001; 21(4):329-34.

34. Fincham JE. Response rates and responsiveness for surveys, standards, and the journal. Am J Pharm Educ. 2008;72(2):43.

35. Linendoll N, Saunders T, Burns R, Nyce JD, Wendell KB, Evens AM, et al. Health-related quality of life in Hodgkin lymphoma: a systematic review. Health Qual Life Outcomes. 2016:14(1):114.

36. Marcellusi A, Capone A, Favato G, Mennini FS, Baio G, Haeussler K, HPV Italian Collaborative Study Group, et al. Health utilities lost and risk factors associated with HPV-induced diseases in men and women: the HPV Italian collaborative study group. Clin Ther. 2015;37(1):156-167.e4.

37. Noel CW, Lee DJ, Kong Q, Xu W, Simpson C, Brown D, et al. Comparison of health state utility measures in patients with head and neck cancer. JAMA Otolaryngol Head Neck Surg. 2015;141(8):696-703.

38. Conway EL, Farmer KC, Lynch WJ, Rees GL, Wain G, Adams J. Quality of life valuations of HPV-associated cancer health states by the general population. Sex Transm Infect. 2012;88(7):517-21.

39. Hamilton DW, Bins JE, McMeekin P, Pedersen A, Steen N, De Soyza A, et al. Quality compared to quantity of life in laryngeal cancer: a time trade-off study. Head Neck. 2016;38(Suppl 1):E631-7.

40. Hollenbeak CS, Lowe VJ, Stack BC Jr. The cost-effectiveness of fluorodeoxyglucose 18-F positron emission tomography in the N0 neck. Cancer. 2001;92(9):2341-8.

41. Jalukar V, Funk GF, Christensen AJ, Karnell LH, Moran PJ. Health states following head and neck cancer treatment: patient, health-care professional, and public perspectives. Head Neck. 1998;20(7):600-8.

42. Ringash J, Redelmeier DA, O'Sullivan B, Bezjak A. Quality of life and utility in irradiated laryngeal cancer patients. Int J Radiat Oncol Biol Phys. 2000;47(4):875-81.

43. Weiss MH, Harrison LB, Isaacs RS. Use of decision analysis in planning a management strategy for the stage N0 neck. Arch Otolaryngol Head Neck Surg. 1994;120(7):699-702.
44. de Almeida JR, Villanueva NL, Moskowitz AJ, Miles BA, Teng MS, Sikora A, et al. Preferences and utilities for health states after treatment for oropharyngeal cancer: transoral robotic surgery versus definitive (chemo)radiotherapy. Head Neck. 2014;36(7):923-33.

45. Llewellyn-Thomas HA, Sutherland HJ, Thiel EC. Do patients' evaluations of a future health state change when they actually enter that state? Med Care. 1993;31(11):1002-12.

46. van der Donk J, Levendag PC, Kuijpers AJ, Roest FH, Habbema JD, Meeuwis CA, et al. Patient participation in clinical decision-making for treatment of T3 laryngeal cancer: a comparison of state and process utilities. J Clin Oncol. 1995:13(9):2369-78.

47. del Barco ME, Mesía R, Adansa Klain JC, Vázquez Fernández S, MartínezGalán J, Pastor Borgoñon M. Et al; Spanish head and neck cancer cooperative group (TTCC). Phase II study of panitumumab and paclitaxel as first-line treatment in recurrent or metastatic head and neck cancer. TTCC2009-03/NECTITAX study. Oral Oncol. 2016;62:54-9.

48. Ouattassi N, Benmansour N, El Fakir S, Nejjari C, Alami MN. Translation and validation of EORTC QLQ-H\&N 35 into Moroccan Arabic for ENT head and neck cancer patients in Morocco. Eur Arch Otorhinolaryngol. 2016;273(9): 2727-34.

49. Parrilla C, Minni A, Bogaardt H, Macri GF, Battista M, Roukos R, et al. Pulmonary rehabilitation after Total Laryngectomy: a multicenter time-series clinical trial evaluating the Provox XtraHME in HME-Naïve patients. Ann Otol Rhinol Laryngol. 2015;124(9):706-13.

50. Rogers SN, Miller RD, Ali K, Minhas AB, Williams HF, Lowe D. Patients' perceived health status following primary surgery for oral and oropharyngeal cancer. Int J Oral Maxillofac Surg. 2006;35(10):913-9.

51. Ramaekers BL, Joore MA, Grutters JP, van den Ende P. Jong Jd, Houben R, et al. the impact of late treatment-toxicity on generic health-related quality of life in head and neck cancer patients after radiotherapy. Oral Oncol. 2011:47(8):768-74.

52. Pickard AS, Jiang R, Lin HW, Rosenbloom S, Cella D. Using patient-reported outcomes to compare relative burden of cancer: EQ-5D and functional assessment of cancer therapy-general in eleven types of cancer. Clin Ther. 2016:38(4):769-77.

53. Truong MT, Zhang Q, Rosenthal DI, List M, Axelrod R, Sherman E, et al. Quality of life and performance status from a sub-study conducted within a prospective phase 3 randomized trial of concurrent accelerated radiation plus cisplatin with or without cetuximab for locally advanced head and neck carcinoma: NRG oncology radiation therapy oncology group 0522. Int J Radiat Oncol Biol Phys. 2017;97(4):687-99.

54. Govers TM, Schreuder WH, Klop WM, Grutters JP, Rovers MM, Merkx MA, et al. Quality of life after different procedures for regional control in oral cancer patients: cross-sectional survey. Clin Otolaryngol. 2016; 41(3):228-33

55. Pottel L, Lycke M, Boterberg T, Pottel H, Goethals L, Duprez F, et al. G-8 indicates overall and quality-adjusted survival in older head and neck cancer patients treated with curative radio-chemotherapy. BMC Cancer. 2015;15:875

56. Aro K, Bäck L, Loimu V, Saarilahti K, Rogers S, Sintonen $H$, et al. Trends in the $15 \mathrm{D}$ health-related quality of life over the first year following diagnosis of head and neck cancer. Eur Arch Otorhinolaryngol. 2016:273(8):2141-50.

57. Loimu V, Mäkitie AA, Bäck LJ, Sintonen H, Räsänen P, Roine R, et al. Healthrelated quality of life of head and neck cancer patients with successful oncological treatment. Eur Arch Otorhinolaryngol. 2015;272(9):2415-23.

58. Higgins KM. What treatment for early-stage glottic carcinoma among adult patients: CO2 endolaryngeal laser excision versus standard fractionated external beam radiation is superior in terms of cost utility? Laryngoscope. 2011:121(1):116-34.

59. Kent EE, Ambs A, Mitchell SA, Clauser SB, Smith AW, Hays RD. Health-related quality of life in older adult survivors of selected cancers: data from the SEER-MHOS linkage. Cancer. 2015;121(5):758-65.

60. Chan KK, Willan AR, Gupta M, Pullenayegum E. Underestimation of uncertainties in health utilities derived from mapping algorithms involving health-related quality-of-life measures: statistical explanations and potential remedies. Med Decis Mak. 2014;34(7):863-72.

61. Parthan A, Posner MR, Brammer C, Beltran P, Jansen JP. Cost utility of docetaxe as induction chemotherapy followed by chemoradiation in locally advanced squamous cell carcinoma of the head and neck. Head Neck. 2009;31(10):1255-62

62. Yong JH, Beca J, O'Sullivan B, Huang SH, McGowan T, Warde P, et al. Costeffectiveness of intensity-modulated radiotherapy in oropharyngeal cancer. Clin Oncol (R Coll Radiol). 2012;24(7):532-8. 
63. Krabbe PF, Peerenboom L, Langenhoff BS, Ruers TJ. Responsiveness of the generic EQ-5D summary measure compared to the disease-specific EORTC QLQ C-30. Qual Life Res. 2004;13(7):1247-53.

64. Nichol MB, Sengupta N, Globe DR. Evaluating quality-adjusted life years: estimation of the health utility index (HUI2) from the SF-36. Med Decis Mak. 2001;21(2):105-12.

65. Pow EH, Kwong DL, McMillan AS, Wong MC, Sham JS, Leung LH, et al. Xerostomia and quality of life after intensity-modulated radiotherapy vs. conventional radiotherapy for early-stage nasopharyngeal carcinoma: initial report on a randomized controlled clinical trial. Int J Radiat Oncol Biol Phys. 2006;66(4):981-91.

66. Vermorken JB, Remenar E, van Herpen C, Gorlia T, Mesia R, Degardin M; EORTC 24971/TAX 323 Study Group, et al, Cisplatin, fluorouracil, and docetaxel in unresectable head and neck cancer. N Engl J Med. 2007; 357(17):1695-1704

67. Heiskanen J, Tolppanen AM, Roine RP, Hartikainen J, Hippeläinen M, Miettinen $\mathrm{H}$, et al. Comparison of EQ-5D and 15D instruments for assessing the health-related quality of life in cardiac surgery patients. Eur Heart J Qual Care Clin Outcomes. 2016;2(3):193-200.

68. Vainiola T, Pettilä V, Roine RP, Räsänen P, Rissanen AM, Sintonen $H$. Comparison of two utility instruments, the EQ-5D and the 15D, in the critical care setting. Intensive Care Med. 2010;36(12):2090-3.

69. Lunde L. Can EQ-5D and 15D be used interchangeably in economic evaluations? Assessing quality of life in post-stroke patients. Eur J Health Econ. 2013;14:539-50.

70. Vartiainen P, Mäntyselkä P, Heiskanen T, Hagelberg N, Mustola S, Forssell H, et al. Validation of EQ-5D and 15D in the assessment of health-related quality of life in chronic pain. Pain. 2017;158(8):1577-85.

71. Arnold D, Girling A, Stevens A, Lilford R. Comparison of direct and indirect methods of estimating health state utilities for resource allocation: review and empirical analysis. BMJ. 2009;339:b2688.

72. Dennett SL, Boye KS, Yurgin NR. The impact of body weight on patient utilities with or without type 2 diabetes: a review of the medical literature. Value Health. 2008;11(3):478-86.

73. McDonough CM, Tosteson AN. Measuring preferences for cost-utility analysis: how choice of method may influence decision-making. Pharmaco Economics. 2007;25(2):93-106.

74. Brazier J, Rowen D. NICE DSU Technical Support Document 11: Alternatives to EQ-5D for Generating Health State Utility Values [Internet]. In: NICE Decision Support Unit Technical Support Documents. London: National Institute for Health and Care Excellence (NICE); 2011.

75. Husereau D, Drummond M, Petrou S, Carswell C, Moher D, Greenberg D, et al. Consolidated health economic evaluation reporting standards (CHEERS) —explanation and elaboration: a report of the ISPOR health economic evaluation publication guidelines good reporting practices task force. Value Health. 2013;16:231-50.

76. Meregaglia M, Cairns J. Economic evaluations of follow-up strategies for cancer survivors: a systematic review and quality appraisal of the literature. Expert Rev Pharmacoecon Outcomes Res. 2015;15(6):913-29.

77. National Institute for Health and Care Excellence. Position Statement on use of the EQ-5D-5L valuation set. Available at: https://www.nice.org.uk/Media/ Default/About/what-we-do/NICE-guidance/NICE-technology-appraisalguidance/eq5d5I_nice_position_statement.pdf [Accessed 10 Aug 2017].

78. Fayers PM, Aaronson NK, Bjordal K, Groenvold M, Curran D, Bottomley A, on behalf of the EORTC Quality of Life Group: The EORTC QLQ-C30 scoring manual, $3^{\text {rd }}$ edition. European Organization for Research and Treatment of Cancer (EORTC), Brussels (2001).

79. Weitzner MA, Meyers CA, Gelke CK, Byrne KS, Cella DF, Levin VA. The functional assessment of cancer therapy (FACT) scale. Development of a brain subscale and revalidation of the general version (FACT-G) in patients with primary brain tumors. Cancer. 1995:75(5):1151-61.

\section{Submit your next manuscript to BioMed Central and we will help you at every step:}

- We accept pre-submission inquiries

- Our selector tool helps you to find the most relevant journal

- We provide round the clock customer support

- Convenient online submission

- Thorough peer review

- Inclusion in PubMed and all major indexing services

- Maximum visibility for your research

Submit your manuscript at www.biomedcentral.com/submit
Biomed Central 